\title{
Levantamento epidemiológico em saúde bucal como recurso didático-pedagógico na formação clínico-epidemiológica do cirurgião-dentista
}

\author{
Manoelito Ferreira Silva-Junior*; Veridiane Gemelli Christ**; Gabriela Fernanda Schiochet**; Vitória \\ Monteiro***; Cristina Berger Fadel**** \\ * Professor Colaborador Adjunto, Universidade Estadual de \\ Ponta Grossa \\ ** Graduanda em Odontologia, Universidade Estadual de \\ Ponta Grossa \\ *** Doutoranda em Odontologia, Universidade Estadual de \\ Ponta Grossa \\ ***** Professora Associada, Departamento de Odontologia, \\ Universidade Estadual de Ponta Grossa
}

Recebido: 25/06/2020. Aprovado: 24/09/2020.

\begin{abstract}
RESUMO
O objetivo do estudo foi relatar o uso do levantamento epidemiológico em saúde bucal como recurso didático-pedagógico na formação clínico-epidemiológica do cirurgião-dentista. Dentro dos objetivos do estágio supervisionado extramuros do Curso de Odontologia da Universidade Estadual de Ponta Grossa, os acadêmicos têm desenvolvido conjuntamente com as Equipes de Saúde Bucal levantamentos epidemiológicos de doenças e/ou necessidades bucais, principalmente para cárie dental, câncer bucal e prótese dentária, segundo o ciclo de vida, em diversos espaços sociais, como os centros de educação infantil, escolas, grupos educativos das Unidades de Saúde, dentre outros. A realização de levantamentos epidemiológicos além de oportunizar os acadêmicos como membros executores e na associação do conteúdo teórico do eixo de Saúde Coletiva na vivência da Atenção Primária à Saúde, como forma de planejamento estratégico situacional a partir dos dados coletados, no monitoramento dos agravos bucais ao longo dos anos em grupos específicos, e na realização de devolutivas para os serviços e para a comunidade, por meio de relatórios, atividades educativas e encaminhamentos e organização da demanda, pode atingir outros objetivos. O foco do relato foi destacar o uso dos levantamentos para aprender sobre o diagnóstico clínico individual e comunitário, e possibilitar aspectos do eixo clínico do curso, como diferenciação das características anatômicas de dentes decíduos e permanentes, diagnóstico diferencial sobre lesões dentárias de mancha branca, alterações e/ou lesões dentárias ou de tecidos moles menos frequentes, dentre outros. Diante sua grande aplicabilidade para além do ensino em Saúde Coletiva, o levantamento epidemiológico em saúde bucal tem sido um recurso didático-pedagógico promissor na formação clínico-epidemiológica do cirurgião-dentista, e sua realização deve ser incentivada nos cursos de Odontologia.
\end{abstract}

Descritores: Inquéritos Epidemiológicos. Serviços de Integração Docente-Assistencial. Materiais de Ensino. Capacitação Profissional. Odontologia. 


\section{INTRODUÇÃO}

Os levantamentos epidemiológicos em saúde bucal têm sido largamente utilizados para a avaliação das condições bucais em populações específicas, seja em nível local ou mundial. As finalidades principais dos levantamentos têm sido determinar a situação atual e monitorar o padrão das doenças ou eventos relacionados à saúde bucal, possibilitar a comparação entre populações, como também de servir de subsídio para o planejamento de políticas públicas ou avaliar o efeito de medidas sanitárias $^{1,2}$.

Diversas condições bucais podem ser avaliadas nos levantamentos epidemiológicos, sendo a cárie dentária coronária ou radicular, necessidade de tratamento para cárie, doença periodontal, uso ou necessidade de prótese dentária, má-oclusão e fluorose dentária as mais avaliadas $^{1,3}$. A escolha das condições bucais mensuradas e dos índices e indicadores a serem utilizados decorre, principalmente, da própria epidemiologia conforme os ciclos de vida ${ }^{1,4}$. No entanto, alterações metodológicas podem ser realizadas para objetivos específicos, como por exemplo, fazendo a extensão da faixa etária estudada ou local de coleta dos dados, ou ainda, pela sua finalidade, como por exemplo o screening (rastreamento), levantamento de necessidade e/ou organização da demanda ${ }^{4,5}$.

Os diagnósticos

comunitário

(epidemiológico) e clínico apresentam diferenças e particularidades que precisam ser destacadas. $\mathrm{O}$ diagnóstico clínico é utilizado para detecção de patologias de forma individual e conta com a presença de diversos recursos. A finalidade principal é o planejamento do tratamento para o paciente avaliado ${ }^{1,6}$. Já o diagnóstico comunitário, apesar de operar por meio de uma abordagem individual, tem a finalidade de identificar a ocorrência e a distribuição de patologias bucais em uma população, podendo, a partir dos dados coletados, subsidiar o planejamento e a execução de ações de saúde bucal com abordagens individuais e coletivas ${ }^{1,3,7}$. Sendo assim, os recursos diagnósticos na dimensão comunitária precisam ser factíveis, reprodutíveis e de baixo custo $^{3,7}$.

No Brasil, houve expansão considerável na oferta de serviço odontológico no setor público de saúde nos últimos anos. No entanto, há uma superposição entre diferentes modelos de atenção à saúde, principalmente pelo modelo hegemônico (biomédico) e o modelo contra-hegemônico (sanitarista). Enquanto o primeiro estabelece a prática individual centrada no profissional e na clínica, lidando com as doenças por meio do diagnóstico, cura e reabilitação, o segundo alicerçase na prática epidemiológica, atuando na prevenção de doenças e na promoção em saúde como forma de cuidado ${ }^{7}$.

A Atenção Primária à Saúde (APS), principalmente no modelo Estratégia Saúde da Família (ESF) tem sido uma forma de atuação em um modelo contra-hegemônico ${ }^{7}$. A APS, além de ser o primeiro nível de atenção à saúde, coordena o cuidado dos diferentes setores e realiza ações compartilhadas sobre os territórios. Neste sentido, a atenção contínua e longitudinal a uma determinada população adscrita torna-se fundamental para o planejamento de ações ${ }^{8}$, sendo os levantamentos epidemiológicos indispensáveis ${ }^{1}$.

As Equipes de Saúde Bucal (ESB) no Sistema Único de Saúde (SUS), que são formadas por poucos profissionais, atuam e se responsabilizam por demandas populacionais aumentadas quando comparadas às equipes médicas, gerando um descompasso entre a capacidade instalada no SUS e um trabalho adequado no campo individual e coletivo ${ }^{9}$.

Nos últimos anos, o país tem intensificado a articulação ensino-serviço-comunidade, principalmente com os serviços da APS, no intuito de atender às Diretrizes Curriculares Nacionais para os cursos de graduação em Odontologia no 
propósito de uma formação profissional generalista, capaz de responder às necessidades de saúde da população na lógica do sistema de saúde vigente, o SUS ${ }^{10}$. Sendo assim, a APS tornou-se um local de aprendizagem que recebe recursos humanos advindos das instituições de ensino superior.

Apesar do ensino relacionado à realização de levantamento epidemiológico em saúde bucal ser uma rotina nas disciplinas do eixo de Saúde Coletiva, principalmente nos estágios supervisionados extramuros, na maioria dos cursos de graduação em Odontologia no Brasil essa prática é voltada tradicionalmente ao ensino das técnicas epidemiológicas envolvidas neste processo $^{10-13}$. Apesar de posta a importância dos levantamentos epidemiológicos como meio de diagnóstico comunitário em saúde e ferramenta fundamental na execução do trabalho no SUS, a interlocução dos acadêmicos de Odontologia nesta prática, além de beneficiar diretamente o serviço e o aprendizado epidemiológico, quando bem delineado, pode ser uma ferramenta fundamental no ensino do eixo clínico em Odontologia ${ }^{11}$.

Nesse contexto, o objetivo do estudo foi relatar o uso do levantamento epidemiológico de saúde bucal como recurso didático-pedagógico na formação clínico-epidemiológica do cirurgiãodentista.

\section{RELATO DA EXPERIÊNCIA}

Trata-se da exposição do curso de Odontologia da Universidade Estadual de Ponta Grossa, Brasil. Em seu Projeto Político Pedagógico, vigente desde 2016, o curso apresenta as disciplinas afetas ao âmbito da Saúde Bucal Coletiva (Saúde Coletiva I e II) integralizadas no segundo ano, distribuídas em um semestre teórico e um semestre de estágio supervisionado extramuros. Durante o estágio, com carga-horária total de 51 horas, há imersão do estudante na Rede de Atenção em Saúde Bucal do município, com exposição aos serviços de saúde de acordo com níveis hierárquicos e execução de atividades articuladas com as ESB na APS.

A proposta pedagógica de orientação para o processo de ensino e aprendizagem da referida disciplina de estágio supervisionado extramuros assume as seguintes categorias: balizamento das concepções teóricas dos alunos, condições objetivas do trabalho em campo com desenvolvimento de práticas significativas em Saúde Coletiva e práxis educativa criadora, na direção de uma educação emancipatória. No campo dos recursos teóricos o que prevalece é o ensaio da ruptura de versões tecnicistas e pragmáticas do agir em saúde, com reforço das práticas de humanização. Os conteúdos de planejamento em saúde e todo o arcabouço do processo de trabalho no SUS completam a conjectura da integração ensino, serviço e comunidade $^{13,14}$.

Neste sentido, ainda que inicialmente permaneçam divisões organizativas tradicionais, nas quais os docentes direcionam o pensar em saúde, assim que os discentes assumem seus postos nos campos de estágio o protagonismo da disciplina passa a ser compartilhado entre alunos, preceptores e comunidade, tornando o estágio uma experiência ao mesmo tempo aberta e direcionada.

As práticas acadêmicas significativas são voltadas para atividades de prevenção e promoção de saúde, sem cunho assistencial direto, ou seja, atividades clínicas individuais na Unidade de Saúde. A partir do ano de 2017, a disciplina de estágio instituiu o desenvolvimento de Projetos de Intervenção (PI) por Unidades de Saúde, com o objetivo de fortalecer os vínculos interinstitucionais. Os PI são elaborados de forma conjunta entre acadêmicos, docentes e equipes de saúde, considerando a realidade local do estágio e a governabilidade e logística de implantação ${ }^{14}$.

Considerando os referidos projetos de 
intervenção, mas não se limitando a eles, os levantamentos epidemiológicos em saúde bucal realizados pelas disciplinas de Saúde Coletiva têm sido vivenciados como importante recurso didático-pedagógico para a formação do acadêmico de Odontologia. Desta forma, diversos levantamentos epidemiológicos se desenvolvem de acordo com as necessidades expressas pelas equipes de saúde bucal, em diferentes espaços sociais do território, como Centros Municipais de Educação Infantil (CMEI) e escolas, na própria Unidade de Saúde, junto a grupos educativos como de gestantes, de idosos e de hipertensos e diabéticos (HIPERDIA).

Ainda, conforme a realidade local há incorporação de levantamentos sobre as principais doenças e/ou necessidades odontológicas de acordo com a faixa etária dos grupos a serem analisados, seguindo a epidemiologia, os critérios e índices da Organização Mundial de Saúde (OMS) ${ }^{15}$ ou versões adaptadas e simplificadas, uma vez que os casos serão encaminhados para a Unidade de Saúde, local com mais recursos para o diagnóstico mais preciso (figura 1 ).

\begin{tabular}{|c|c|c|}
\hline Grupo & Índice & Atividade \\
\hline Pré-escolares & Índice ceo- $\mathrm{d}^{*}$ & $\begin{array}{c}\text { Atividade Educativa } \\
\text { Relatório para CMEI } \\
\text { Relatório para UBS/USF } \\
\text { Encaminhamento para UBS/USF }\end{array}$ \\
\hline Escolares & Índices ceo-d* e CPO-D** & $\begin{array}{c}\text { Atividade Educativa } \\
\text { Relatório para Escola } \\
\text { Relatório para UBS/USF } \\
\text { Encaminhamento para UBS/USF }\end{array}$ \\
\hline Grupo de gestantes & Índice CPO-D** & $\begin{array}{c}\text { Atividade Educativa } \\
\text { Encaminhamento para UBS/USF }\end{array}$ \\
\hline $\begin{array}{l}\text { Grupos HIPERDIA } \\
\text { ou Idosos }\end{array}$ & $\begin{array}{l}\text { Necessidade de prótese dentária } \\
\text { Câncer bucal }\end{array}$ & $\begin{array}{c}\text { Atividade Educativa } \\
\text { Encaminhamento para UBS/USF }\end{array}$ \\
\hline
\end{tabular}

Figura 1. Índices e atividades desenvolvidas por ciclos de vida ou grupos educativos

*Índice ceo-d (índice de dentes decíduos cariados, com indicação de exodontia e obturados);

**Índice CPO-D (Índice de dentes permanentes cariados, perdidos e obturados).

A escolha dos indicadores e/ou índices para avaliação da ocorrência e/ou distribuição de doenças bucais nos levantamentos epidemiológicos realizados dependem da finalidade/objetivo e as especificidades de cada PI. No entanto, em todos os casos há uma devolutiva por meio da realização de ações educativas e/ou preventivas determinadas de forma articulada e conjunta pelos professores, acadêmicos e equipes de saúde. Na definição do PI, é realizado um planejamento estratégico dos problemas existentes nos territórios de atuação do estágio. Esse planejamento visa não apenas conhecer os doentes ou não doentes, e sim estabelecer as principais necessidades em saúde bucal da população analisada e organização da demanda.

Além disto, deixa-se claro que todos os PI tem uma etapa avaliativa do processo, e inclui aspectos de devolutivas como mencionado anteriormente, seja diretamente ao indivíduo participante, como por exemplo, encaminhamentos dos pacientes com necessidades odontológicas, execução de atividades educativas para os grupos vulneráveis, mas também para as instituições 
parceiras, por meio de relatório para os serviços envolvidos, como os centros de ensino infantil, escolas e Unidades de Saúde.

\section{DISCUSSÃO}

O presente relato teve como principal objetivo demonstrar a experiência exitosa da aplicabilidade do levantamento epidemiológico com finalidade mais amplas de formação em Odontologia durante o estágio supervisionado extramuros. Neste viés, o seu uso como recurso didático-pedagógico não se limitou ao ensino no eixo de Saúde Coletiva, mas também no eixo clínico, ou seja, com vistas à formação clínicoepidemiológica do cirurgião-dentista.

Os levantamentos podem ser usados para potencializar o espaço de interlocução entre ensino-serviço-comunidade, e assim contribuir com o aprimoramento profissional e interprofissional $^{16}$, e uma formação acadêmica condizente com as necessidades dos serviços de saúde $^{10}$. Apesar do presente relato estar ligado aos estágios obrigatórios extramurais, deixa-se claro que os levantamentos podem ser realizados de forma independente em outros momentos oportunos ou em outras disciplinas. No entanto, independente da sua finalidade ou objetivo, devese ter uma preocupação adicional no cuidado em relação à execução de devolutivas ${ }^{17}$, por exemplo, encaminhamento dos pacientes com necessidades odontológicas, execução de atividades educativas para os grupos vulneráveis e um relatório para os serviços envolvidos. Os dados coletados compreendem uma ferramenta fundamental na estratégia de vigilância em saúde ${ }^{4}$ e são informações imprescindíveis para o planejamento de ações em saúde. Por isso, acordos prévios devem ser estabelecidos quanto às responsabilidades dos atores envolvidos, por exemplo, encaminhamento ao SUS e/ou às clínicas das universidades.

O levantamento epidemiológico é um método de diagnóstico coletivo, passando de uma visão isolada de caso a caso, para uma visão epidemiológica do todo ${ }^{3,7}$. A lógica de avaliação de saúde bucal fora da clínica das instituições de ensino superior passa a ter um valor profissional importante, porque compreende um momento de efetivação do conhecimento sobre planejamento. Esta etapa passa desde a busca da articulação intersetorial ou com os serviços de saúde, escolha de grupos prioritários, escolha dos indicadores ou índices a serem utilizados, até os recursos humanos, financeiros, materiais e/ou estruturais necessários para execução ${ }^{18}$.

Como na Instituição de Ensino Superior analisada o Projeto Político Pedagógico no campo da saúde coletiva é dividido em um semestre teórico e um semestre de estágio extramuros na atenção primária, para que seja possível estabelecer um conhecimento teórico-prático de qualidade, acredita-se que no momento da realização dos levantamentos epidemiológicos exista uma transposição da teoria à prática ${ }^{14}$. A aprendizagem por meio de atividades práticas amplia a obtenção de conhecimento e interesse por parte do graduando, e tem se mostrado mais eficiente em relação a metodologias usualmente utilizadas, as quais são limitadas ao plano cognitivo; visto que as ações a serem desenvolvidas exigem o conhecimento prévio do assunto, e com isso, as experiências são mais rentáveis e significativas ${ }^{19}$.

A sistemática tradicional de ensino ou o formato de ensino fragmentado das disciplinas do eixo de saúde coletiva e do eixo clínico ainda representam uma barreira na formação do profissional da saúde. O uso de metodologias ativas é uma alternativa viável que possibilita maior assimilação teórico-prática do conteúdo proposto, e pode preencher essa lacuna existente entre o ensino e a aprendizagem ${ }^{20}$. A forma facilitadora de aprendizado possibilita que o aluno adquira conhecimentos práticos em ambiente específico, e reforce a abordagem teórica trabalhada 
previamente em classe. Desse modo, ocorre maior interação entre ensino, serviço e comunidade ${ }^{10-13}$. Ainda há possibilidade de maior assimilação do conteúdo por parte do estudante e a construção integral do processo de desenvolvimento do profissional de saúde, e assim, ser mais responsivo as diversas demanda da sociedade em uma formação integral ${ }^{20}$.

Para demonstrar as implicações no ensino de eixo clínico, podemos relatar alguns exemplos, dentre eles, a diferenciação entre dentes decíduos e dentes permanentes. Na população de escolares pode ser necessário o uso híbrido dos índices de dentes decíduos cariados, exodontia indicada e perdidos (ceo-d) e de dentes permanentes cariados, perdidos e obturados (CPO-D) simultaneamente ${ }^{21}$. Durante a execução do levantamento epidemiológico, a observação ampla de um grande número de pessoas, em curto período de tempo, possibilita a percepção da diferenciação da anatomia de dentes decíduos e permanentes. Em situação rotineira, dentro da instituição de ensino, o atendimento odontológico clínico individual seria limitado ao contato com apenas um ou dois pacientes por período, o que pode limitar a observação da dentição em diferentes faixas etárias em um mesmo dia e um total limitado no semestre. Apesar das diversas características anatômicas teoricamente conhecidas pelos acadêmicos, ainda ocorre dificuldade em diferenciar os dentes decíduos e permanentes, principalmente na fase de dentadura mista. $\mathrm{O}$ contato com maior número de escolares com dentadura mista e em diferentes estágios de erupção dentária, representa um ponto positivo do uso dessa metodologia ativa de aprendizado, que visa articular a teoria e a prática no momento do processo de ensinoaprendizagem $^{22}$.

A medida que aumenta a proporção de crianças e adolescentes livres de cárie no Brasil e no mundo ${ }^{2}$, e a consequente redução dos índices ceo-d e CPO-D ${ }^{23}$, torna-se cada vez mais rotineira a avaliação de alterações dos tecidos dentários com características clínicas semelhantes à cárie dentária. Os levantamentos epidemiológicos podem ser, inclusive, utilizados como recurso no ensino do diagnóstico diferencial de manchas brancas, como por exemplo, hipoplasia, hipomineralização molar-incisivo (HMI) e fluorose. Segundo Jälevik, Szigyarto-Matei e Robertson (2019) ${ }^{24}$, profissionais treinados possuem maior capacidade para diferenciar alterações de desenvolvimento no esmalte de lesões de cárie dentária, quando comparados com profissionais sem treinamento prévio.

Além das alterações ou anomalias das estruturas dentárias, em levantamentos rotineiros há uma probabilidade de visualização de patologias e alterações bucais menos frequentes na rotina clínica das universidades, mas com prevalência significativa na população. Pode-se relatar, por exemplo, anquiloglossia, glossite migratória, dentes supranumerários, morsicatium, baixa inserção de freio labial, mucocele, rânula, fístulas, dentre outras ${ }^{25,26}$. Nestes casos, reforça-se a necessidade contínua de responsabilidade ética e profissional na prestação do serviço, seja na instituição de ensino superior, e principalmente nos espaços sociais de interlocução com os serviços de saúde.

No presente relato ficou evidente que os levantamentos epidemiológicos devem ser realizados para além da avaliação de cárie dentária e em grupos de escolares, como tradicionalmente realizado no Brasil ${ }^{27}$. Apesar dos índices ceo-d e CPO-D, Índice de Alterações Gengivais ou o Índice de Higiene Oral (IHO) serem amplamente utilizados, podem ser pouco aplicáveis a algumas faixas etárias ou ainda ser inadequados para alguns casos. Sendo assim, pode haver a necessidade de adaptação ou simplificação, uma vez que a articulação com os serviços de saúde pode garantir um diagnóstico clínico individual a posteriori. Além disso, outros indicadores ou índices podem 
ser aplicados no planejamento de ações em saúde bucal do território analisado conforme as características da população de interesse $\mathrm{e}^{28}$.

O ensino de habilidades e competências de planejamento e gestão em saúde nos cursos de graduação em Odontologia permite que o aluno tenha vivência da atuação profissional em saúde bucal, tanto em atividade clínica, quanto no gerenciamento das ações em saúde pela equipe odontológica. Além disso, o convívio com o ambiente físico e com os usuários do SUS contribui para a formação de um profissional crítico e autoavaliativo $^{29}$. A formação de um profissional com gestão resolutiva e apto a trabalhar em equipe multiprofissional depende de uma formação de qualidade com interação entre ensino-serviçocomunidade $^{23,30}$.

A falta de estudos que se dediquem a utilizar o levantamento epidemiológico em saúde bucal como recurso didático-pedagógico no ensino em Odontologia representa uma limitação para comparação com o atual estudo, mostrando que a temática ainda é pouco explorada ${ }^{11,12}$. Além disso, o relato de experiência é subjetivo ao conhecimento e aprendizagem dos participantes específicos da instituição e, por isso, pode ter uma utilização ou natureza diferente em outras realidades. Adaptações frente ao exposto no presente relato podem ser necessárias para atender aos objetivos educacionais de cada instituição e para o processo de aprimoramento e desenvolvimento de estratégias de aprendizagem que, por consequência, contribuam para o fortalecimento da relação ensino-serviço-comunidade.

Com este relato, entende-se que a execução de um levantamento epidemiológico de forma delineada e articulada junto aos serviços de saúde em espaços sociais, pode trazer benefícios para todos os agentes envolvidos. Para o serviço, auxilia no diagnóstico situacional de saúde e na determinação de grupos vulneráveis e critérios de prioridade no atendimento; para a comunidade, resulta em uma atuação mais qualificada e responsiva conforme suas necessidades, inclusive com otimização do acesso e acolhimento aos serviços de saúde. Para o ensino, como descrito no foco do presente relato, complementa-se o uso dos levantamentos epidemiológico como recurso de ensino-aprendizagem para além do ensino teórico em Saúde Coletiva, e mais especificamente de Epidemiologia, mas uma chance oportuna para o ensino do eixo de disciplinas do eixo clínico dos cursos de Odontologia, como Cariologia, Periodontia, Estomatologia, Prótese Dental, dentre outras especialidades odontológicas.

\section{CONSIDERAÇÕES FINAIS}

O presente relato mostrou como a integração ensino-serviço-comunidade, na disciplina de estágio supervisionado extramuros, tem favorecido a realização de levantamentos epidemiológicos com finalidade para além do ensino de natureza epidemiológica, mas também facilidade do ensino do eixo clínico. Sendo assim, além da aprendizagem sobre a operacionalização de um diagnóstico comunitário, planejamento e organização da demanda, pode auxiliar na formação do eixo clínico, como por exemplo, sobre a diferenciação de dentes decíduos e permanentes, diagnóstico diferencial de estruturas dentárias, como no caso de manchas brancas, ou na identificação de alterações dentárias e/ou de tecidos moles, dentre outros.

Nesse sentido, é um recurso que possibilita atendar às Diretrizes Curriculares Nacionais, quando há um delineamento adequado no desenvolvimento das atividades a serem executadas, e de forma articulada com os serviços. $\mathrm{O}$ relato demonstra que a atividade proposta se constitui em ferramenta didático-pedagógica promissora na integração entre os eixos de saúde coletiva e clínico, no intuito de uma formação generalista com perfil profissional formado em uma lógica clínico-epidemiológica. 
Desse modo, o estágio supervisionado contribui para a formação de um profissional ético, generalista e comum olhar crítico voltado à saúde de toda a população. $O$ processo que envolve desde a fase inicial de discussão dos problemas existentes, avaliação da viabilidade, até a fase de execução da estratégia, com devolutiva à população por meio do desenvolvimento de ações propostas no planejamento em saúde.

\section{ABSTRACT \\ Oral Health Epidemiological Survey as a Didactic-Pedagogical Resource in the Clinical Epidemiology Education of Dental Surgeons}

This study aimed to report the use of an epidemiological survey on oral health as a didactic-pedagogical resource in the initial education of dental surgeons. Following the objectives of the external supervised internship of the Dentistry undergraduate course at the State University of Ponta Grossa, students have developed epidemiological surveys on oral illnesses and/or needs in cooperation with Oral Health Teams. These surveys focus mainly on dental caries, oral cancer and dental prosthesis according to life cycles, in several social spaces such as child daycare centers, schools, education teams in health units, among others. Carrying out epidemiological surveys, in addition to providing students with opportunities of acting as executors, also enables them to associate theoretical content from the collective health axis to the development of Primary Health Care. It provides a model of situational strategic planning from the data collected to monitor oral diseases along the years in specific groups and results in feedback for the services provided and for the community through reports, education activities as well as new strategies and demand organization, and it might also achieve other objectives. The focus of this report was to highlight the use of surveys to learn about individual and community clinical diagnoses, enabling certain aspects of the course clinical axis such as the differentiation of anatomical characteristics of deciduous and permanent teeth, differential diagnosis of white-spot dental lesions, less frequent dental or soft tissue alterations and/or lesions, among others. Due to their applicability beyond the collective health teaching, oral health epidemiological surveys have been seen as a promising didacticpedagogical resource in the clinical epidemiology education of dental surgeons and their use should be fostered in dentistry courses.

Descriptors: Health Surveys. Teaching Care Integration Services. Teaching Materials. Professional Training. Dentistry.

\section{REFERÊNCIAS}

1. Roncalli AG, Silva NN, Nascimento AC, Freitas CHSM, Casotti E, Peres KG, et al. Aspectos metodológicos do Projeto SBBrasil 2010 de interesse para inquéritos nacionais de saúde. Cad Saúde Pública. 2012; 28Suppl. s40-s57.

2. Kassebaum NJ, Smith AGC, Bernabé E, Fleming TD, Reynolds AE, Vos T, et al. GBD 2015 Oral Health Collaborators. Global, regional, and national prevalence, incidence, and disability-adjusted life years for oral conditions for 195 countries, 19902015: a systematic analysis for the global burden of diseases, Injuries, and Risk Factors. J Dent Res. 2017; 96(4):380-7.

3. Roncalli AG, Côrtes MIS, Peres KG. Perfis epidemiológicos de saúde bucal no Brasil e os modelos de vigilância. Cad Saúde Pública. 2012; 28 Sup: s58-s68.

4. Soares FF, Freire MCM, Reis SCGB. The 2010 Brazilian Oral Health Survey (SBBrasil 2010 Project): what do the coordinators propose for future surveys? Interface (Botucatu). 2018; 22(64):981-9.

5. Antunes, JL; Peres MA. Epidemiologia da Saúde Bucal. 2 ed. Rio de Janeiro: Guanabara Koogan, 2013; 738 p.

6. Cerri A, Guarim JÁ, Genovese WJ. 
Planejamento e diagnóstico em Odontologia com os princípios bioéticos. Rev Assoc Paul Cir Dent. 2015; 69(3): 216-25.

7. Pereira, Antonio Carlos. Tratado de Saúde Coletiva em Odontologia. 1 ed. Rio de Janeiro: Napoleão, 2009.

8. Pimentel FC, Martelli PJL, Araújo-Junior JLAC, Acioli RML, Macedo CLSV. Análise da atenção à saúde bucal na Estratégia de Saúde da Família do Distrito Sanitário VI, Recife (PE). Ciênc Saúde Colet. 2010; 15(4): 2189-96.

9. Brasil. Ministério da Saúde. Portaria $\mathrm{n}^{\mathrm{o}}$ 2.436, de 21 de setembro de 2017. Aprova a Política Nacional de Atenção Básica, estabelecendo a revisão de diretrizes para a organização da Atenção Básica, no âmbito do Sistema Único de Saúde (SUS). Brasília, DF: Ministério da Saúde, 2020. [internet]. [Acesso em: 30 nov 2017]. Disponível em: http://www.brasilsus.com.br/index.php/legi slacoes/gabinete -do- -ministro/16247portaria-n-2-436-de-21-de-setembro-de2017.

10. Faé JM, Silva-Junior MF, Carvalho RB, Esposti CDD, Pacheco KTS. A integração ensino-serviço em Odontologia no Brasil. Rev ABENO. 2016; 16 (3): 7-18.

11. Lima DC, Pereira AP, Drumond ES. Percepção dos acadêmicos de odontologia da UNIFAL/MG quanto a Formação em Saúde da Família. Rev Odontol Bras Central. 2013; 21(60): 50-5.

12. Fonsêca GS, Junqueira SR, de Araújo ME, Botazzo C. Modelo lógico-ideal para o estágio curricular supervisionado: a educação pelo trabalho na formação odontológica. Rev ABENO. 2015; 15(2):211.

13. Silva-Junior MF, Pacheco KTS, Carvalho RB. Multiplicidade de atuações do acadêmico de Odontologia no estágio curricular: relato de experiência. Arq Odontol. 2015; 51(4): 194-204.

14. Luz MC, Baldani MH, Silva-Junior MF. Integração ensino-serviço-comunidade no curso de odontologia: experiências exitosas entre o município e a universidade. Arch Health Invest. 2018; 7: 84.

15. Oral Health Survey. Basic methods. 5th ed. Geneva, World Health Organization, 2013.

16. Brisolara KF, Gasparini S, Davis AH, Sanne S, Andrieu SC, James J, et al. Supporting health system transformation through an interprofessional education experience focused on population Health. J Interprof Care. 2018; 33; 1-4

17. Souza GC, Lopes MLDS, Roncalli AG, Medeiros-Junior A, Clara-Costa I. Referência e contra referência em saúde bucal: regulação do acesso aos centros de especialidades odontológicas. Rev Salud Pública. 2015; 1(3): 416-28.

18. Brasil, Ministério da Saúde. Série A. Normas e Manuais Técnicos Cadernos de Atenção Básica - n. ${ }^{\circ} 17.1^{\mathrm{a}}$ ed. Brasília: DF; 2008. [Acesso em: 12 set 2020]. Disponível em: https://bvsms.saude.gov.br/bvs/publica coes/saude bucal.pdf.

19. Benito GAV, Tristão KM, Paula ACSF, Santos MA, Ataide LJ, Lima RCD. Desenvolvimento de competências gerais durante o estágio supervisionado. Rev Bras Enferm. 2012; 65(1):172-8.

20. Noro RLA, Farias-Santos BCS, Souza PHS, Pinheiro IAG, Borges REA, Nunes LMF, et al. O professor (ainda) no centro do processo ensino-aprendizagem em Odontologia. Rev ABENO. 2015; 15(1): 2-11.

21. Moraes SNS, Arsenian MB, Tuccil, R. Avaliação clínica e utilização do índice CPO-D/“ceo-d" em crianças da Escola Municipal José Carlos Porto-Paraty/RJ. J Health Sci Inst. 2014; 32(3): 235-40. 
22. Freitas CM, Freitas CASL, Parente JRF, Vasconcelos MISO, Lima GK, Mesquita $\mathrm{KO}$, et al. Uso de metodologias ativas de aprendizagem para a educação na saúde: análise da produção científica. Trab Educ Saúde. 2015; 13 (Suppl. 2): 117-30.

23. Martins RJ; Garbin CAS; Garbin AJI; Moimaz SAS; Saliba O. Declínio da cárie em um município da região noroeste do Estado de São Paulo, Brasil, no período de 1998 a 2004. Cad Saúde Pública. 2006; 22(5):1035-41.

24. Jälevik B, Szigyarto-Matei A, Robertson A. Difficulties in identifying developmental defects of the enamel: a BITA study. Eur Arch Paediatr Dent. 2019; 20(5); 481-8.

25. Neville BW, Damm DD, Allen CM, Bouquot JE. Patologia oral e maxilofacial. 4ed. Rio de Janeiro: Elsevier; 2016. 912p.

26. Amorim CS; Camargo WR. Prevalência das lesões bucais em ações comunitárias odontológicas. BJSCR. 2014; 8(2):19-23.

27. Oliveira AGRC, Unfer B, Costa ICC, Arcieri RM, Guimarães LOC, Saliba NM. Levantamentos epidemiológicos em saúde bucal: análise da metodologia proposta pela Organização Mundial da Saúde. Rev Bras Epidemiol. 1998; 1(2): 177-89.

28. Kleba ME; Krauser IM; Vendruscolo C. O planejamento estratégico situacional no ensino da gestão em saúde da família. Texto Contexto Enferm. 2011; 20(1): 184-93.

29. Santos KT, Ferreira L, Batista RJ, Bitencourt CTF, Araújo RP, Carvalho RB. Percepção discente sobre a influência de estágio extramuro na formação acadêmica odontológica. Rev Odontol. 2013; 42(6): 420-5.

30. Gomes VG. O ensino da gestão no Estágio Curricular Supervisionado II da Odontologia da Universidade Federal do Rio Grande do Sul. Rio Grande do sul. Porto Alegre: Universidade Federal do Rio Grande do Sul. Porto Alegre, 2019. Trabalho de Conclusão de Curso em Odontologia. [Acesso em: 20 maio 2020. Disponível em: https://www.lume.ufrgs.br/bitstream/handle /10183/206087/001112457.pdf?sequence $=1$ \&isAllowed $=\mathrm{y}$.

\section{Correspondência para:}

Manoelito Ferreira Silva Junior

e-mail: manoelito_fsjunior@hotmail.com

Departamento de Odontologia

Campus Uvaranas - Bloco $\mathrm{M}-52 \mathrm{~B}-2^{\circ}$ Piso

Avenida General Carlos Cavalcanti, 4748, Uvaranas

84030-900 Ponta Grossa/PR 